\title{
Sommario
}

Prefazione - VII

\section{Storia sacra}

Nicolangelo D’Acunto

L'ascesi narrata. Varia fortuna delle Vite dei Padri del deserto fra imitazione monastica e penitenza istituzionalizzata -3

Raymund Wilhelm

Bonvesin da la Riva agiografo e volgarizzatore. Dagli exempla della Vita scholastica ai miracoli in volgare -19

Elisa De Roberto

Raccontare il miracolo nel Medioevo italiano. Aspetti pragmatici e testuali della letteratura miracolistica in volgare - 41

Massimo Zaggia

Alle origini della storia sacra: l'avvio del Genesi in volgare italiano -85

Paolo Pellegrini

«Sul cavoge» / «sui cavegi». Nota sul pronome enclitico in italiano antico - 149

Michele Colombo

Lettera e voce nella «Passione Mai» in veneziano antico 155

\section{Storia profana}

Roberta Cella

L'epistola dei palermitani ai messinesi (13 aprile 1282)

e il suo volgarizzamento - 173

Simona Brambilla

Note sul volgarizzamento della Fam. XII 2 di Francesco Petrarca -197

\section{Chiara De Caprio}

Figure dell'autore nei volgarizzamenti e nelle cronache in volgare. Aspetti teorici e linee di una ricerca storico-linguistica nei testi medioevali — 211 
VI - Sommario

\section{Enrico Faini}

Vegezio e Orosio: storia, cavalleria e politica nella Firenze

del tardo Duecento — 237

Cristiano Lorenzi

Tradurre la storia romana. Il caso delle due redazioni del volgarizzamento della prima Catilinaria fra Due- e Trecento — 255

Giulio Vaccaro

Storia e geografia di un centone di volgarizzamenti: il Libro dell'Aquila -273

Luca Barbieri

Materia troiana e materia ovidiana nel ms. Gaddi 71 della Biblioteca Laurenziana di Firenze - 299

Simone Pregnolato

La «verace ystoria». Avviamento allo studio del volgarizzamento troiano di Mazzeo Bellebuoni — 319

\section{Indici}

1 Indice dei nomi - 375

2 Indice dei manoscritti e dei documenti d'archivio - 381 